\title{
The Effects of Hypercapnia on Cerebral Autoregulation in Ventilated Very Low Birth Weight Infants
}

\author{
JEFFREY R. KAISER, C. HEATH GAUSS, AND D. KEITH WILLIAMS \\ Department of Pediatrics [J.R.K.], Division of Neonatology, College of Medicine, Department of \\ Biostatistics [C.H.G.], College of Public Health, and Division of Biostatistics [D.K.W.], College of \\ Medicine, University of Arkansas for Medical Sciences, Little Rock, Arkansas, 72205
}

\begin{abstract}
Permissive hypercapnia, a strategy allowing high $\mathrm{PaCO}_{2}$, is widely used by neonatologists to minimize lung damage in ventilated very low birth weight (VLBW) infants. While hypercapnia increases cerebral blood flow (CBF), its effects on cerebral autoregulation of VLBW infants are unknown. Monitoring of mean $\mathrm{CBF}$ velocity ( $\mathrm{mCBFv}), \mathrm{PaCO}_{2}$, and mean arterial blood pressure (MABP) from 43 ventilated VLBW infants during the first week of life was performed during and after 117 tracheal suctioning procedures. Autoregulation status was determined during tracheal suctioning because it perturbs cerebral and systemic hemodynamics. The slope of the relationship between mCBFv and MABP was estimated when $\mathrm{PaCO}_{2}$ was fixed at 30, $35,40,45,50,55$, and $60 \mathrm{~mm} \mathrm{Hg}$. A slope near or equal to 0 suggests intact autoregulation, i.e. $\mathrm{CBF}$ is not influenced by MABP. Increasing values $>0$ indicate progressively impaired autoregulation. Infants weighed $905 \pm 259 \mathrm{~g}$ and were $26.9 \pm$ 2.3 wk gestation. The autoregulatory slope increased as $\mathrm{PaCO}_{2}$ increased from 30 to $60 \mathrm{~mm} \mathrm{Hg}$. While the slopes for $\mathrm{PaCO}_{2}$ values of 30 to $40 \mathrm{~mm} \mathrm{Hg}$ were not statistically different from 0 , slopes for $\mathrm{PaCO}_{2} \geq 45 \mathrm{~mm} \mathrm{Hg}$ indicated a progressive loss of
\end{abstract}

\section{ABSTRACT}

cerebral autoregulation. The autoregulatory slope increases with increasing $\mathrm{PaCO}_{2}$, suggesting the cerebral circulation becomes progressively pressure passive with hypercapnia. These data raise concerns regarding the use of permissive hypercapnia in ventilated VLBW infants during the first week of life, as impaired autoregulation during this period may be associated with increased vulnerability to brain injury. (Pediatr Res 58: 931935, 2005)
BP, blood pressure
Abbreviations
CBF, cerebral blood flow
DOL, day of life
IVH, intraventricular hemorrhage
MABP, mean arterial blood pressure
mCBFv, mean cerebral blood flow velocity
PRESS, predicted residual sum of squares
PVL, periventricular leukomalacia
VLBW, very low birth weight

Neonatologists frequently use permissive hypercapnia, a strategy that allows high $\mathrm{PaCO}_{2}(45-55 \mathrm{~mm} \mathrm{Hg})$, to avoid ventilator-induced lung injury during mechanical ventilation of premature infants (1). The theoretical benefit of permissive hypercapnia is that lower tidal volumes and mean airway pressures may prevent alveolar overdistension and lung injury (1). Randomized controlled trials of permissive hypercapnia, however, failed to demonstrate significant improvements in survival without chronic lung disease over routine ventilation

Submitted November 22, 2004; accepted March 16, 2005

Correspondence: Jeffrey R. Kaiser, M.D., Arkansas Children's Hospital, 800 Marshall St., \#512, Little Rock, AR 72202; e-mail: kaiserjeffreyr@uams.edu.

J.R.K. was supported by a National Institute of Neurological Disorders and Stroke Grant 1 K23 NS43185. The University of Arkansas for Medical Sciences General Clinical Research Center (M01RR14288) also supported this study. Presented in part at the 2004 Pediatric Academic Society, Society for Pediatric Research meeting, San Francisco, May $1-4,2004$.

DOI: 10.1203/01.pdr.0000182180.80645.0c strategies $(1,2)$. Despite its widespread use, the effects of permissive hypercapnia on cerebral autoregulation of premature infants are not known.

Cerebral autoregulation is an essential physiologic mechanism that maintains constant blood flow to the brain despite variations in cerebral perfusion pressure (3). The BP range over which $\mathrm{CBF}$ remains constant is known as the autoregulatory plateau. In this portion of the curve, $\mathrm{CBF}$ is independent of BP and the slope is 0. Below the autoregulatory plateau, CBF declines; above it, $\mathrm{CBF}$ rises in a pressure-passive manner (Fig. 1 A) (4,5). In contrast, in impaired autoregulation, CBF linearly follows changes in BP (over all BPs), where the slope is significantly $>0$ (Fig. 1B) (6). Despite the widespread belief that cerebral autoregulation is impaired in sick, ventilated premature infants (6), recent evidence using continuous monitoring systems suggests that many premature infants do have intact autoregulation $(7,8)$. 

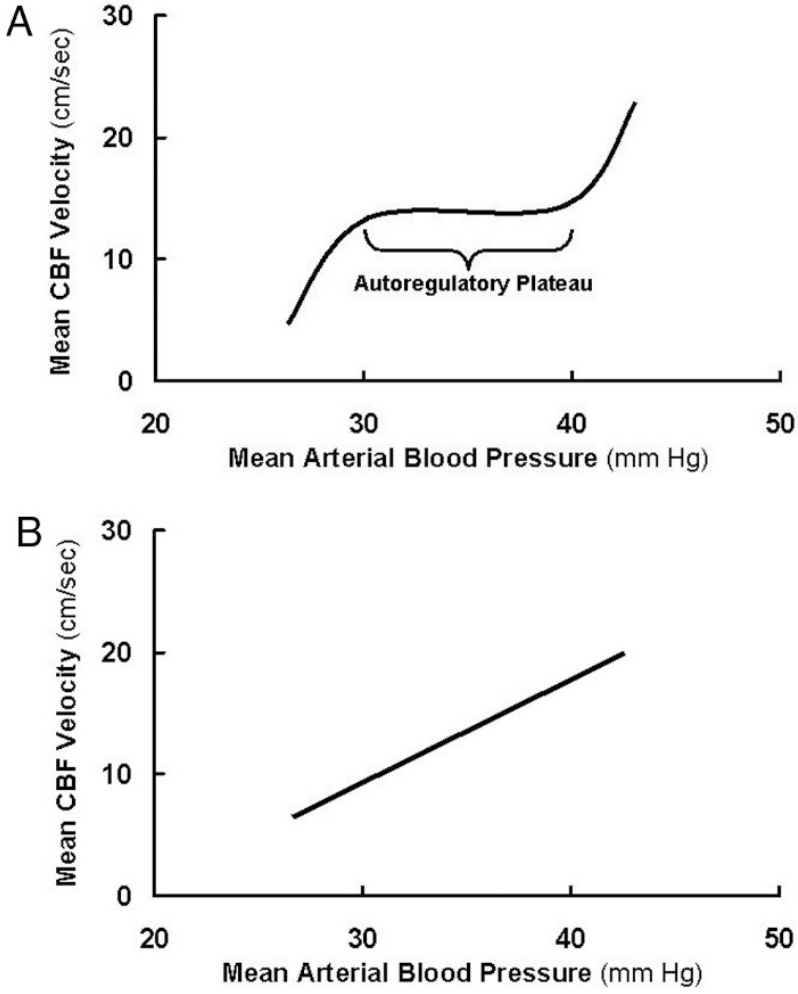

Figure 1. (A) Example of intact cerebral autoregulation. Adapted from van de Bor and Walther (4) and Papile et al. (5). (B) Example of impaired cerebral autoregulation. Adapted from Lou et al. (6).

Hypercapnia causes cerebral vasodilation, increases CBF in premature infants, and may contribute to the development of IVH $(9-12)$. Although the etiology of neonatal brain injury is multifactorial, disturbances of $\mathrm{CBF}$ and cerebral autoregulation play an important role $(7,13,14)$. Hypercapnia in animals has been associated with impaired cerebral autoregulation $(3,15)$. Conversely, impaired autoregulation associated with a variety of pathologic conditions in animals and adults can be restored by hyperventilation $\left(\mathrm{PaCO}_{2} 25-30 \mathrm{~mm} \mathrm{Hg}\right)$ (16). Although the ideal $\mathrm{PaCO}_{2}$ range for premature infants is not known, we hypothesized that cerebral autoregulation would become progressively impaired with increasing $\mathrm{PaCO}_{2}$. Therefore, the objective of this study was to examine the effects of increasing $\mathrm{PaCO}_{2}$ on the cerebral autoregulatory capacity of ventilated premature infants during the first week of life.

\section{METHODS}

Study sample. VLBW (birth weight, 501-1500 g) infants born at the University of Arkansas for Medical Sciences between July 2002 and February 2004 were eligible for this observational study if they required mechanical ventilation for respiratory distress syndrome, had a 3.7 Fr umbilical arterial catheter placed during newborn stabilization, and had normal cranial ultrasound findings before the study. Infants with major congenital anomalies were excluded. Routine intensive care procedures were left to the discretion of the attending neonatologist.

Monitoring equipment. Continuous BP monitoring was performed with an umbilical arterial catheter (Diametrics Medical Ltd., St. Paul, MN; Argyle/ Tyco Healthcare/Kendall, Mansfield, MA) attached to a BP transducer (Transpac IV, Abbott Critical Care Systems, North Chicago, IL). The transducer was connected to a cardiorespiratory monitor (Spacelabs, Redmond, WA) and calibrated.

Continuous blood gas monitoring was performed with a Neotrend-L (Diametrics Medical Ltd.) fiber optic sensor that was placed in the umbilical arterial catheter. The sensor was connected to a satellite Trendcare monitor (Diametrics Medical Ltd.) that continuously displayed $\mathrm{pH}, \mathrm{PaCO}_{2}, \mathrm{PaO}_{2}$, temperature, bicarbonate, base excess, and oxygen saturations in real-time.

Continuous measurements of right middle cerebral artery CBF velocity were made using a transcranial Doppler ultrasound system (Nicolet Biomedical Pioneer, Madison, WI). A lightweight 2-MHz pulsed-wave button transducer was placed transtemporally anterior to the external ear and above the zygomatic arch and held in place by an appropriately sized crocheted hat (courtesy of the Arkansas Extension Homemakers Council). A depth of 16-22 mm was used to study the M1 portion of the middle cerebral artery. A $100-\mathrm{Hz}$ low-pass filter was used to dampen "noise" from the vessel wall. Reliable CBF velocity measurements were obtained when the highest intensity acoustic signal was perceived and the highest intensity Doppler spectra visualized. The ultrasound intensity $\left(5-21 \mathrm{~mW} / \mathrm{cm}^{2}\right)$ was well below the recommendations for continuous monitoring (17). CBF velocity tracings were consistent $>1 \mathrm{~h}$ with minimal or no drift in the signal intensity. The investigator or research assistant was present at the bedside during all monitoring periods.

Analog signals from the BP monitor (112 samples $/ \mathrm{s}, 10 \mathrm{mV} / 1 \mathrm{~mm} \mathrm{Hg}$ ), blood gas monitor (1 sample/s; $\mathrm{PaCO}_{2}: 25 \mathrm{mV} / 1 \mathrm{~mm} \mathrm{Hg}$; $\mathrm{PaO}_{2}: 4 \mathrm{mV} / 1 \mathrm{~mm}$ $\mathrm{Hg}$ ), and transcranial Doppler (100 samples/s, $10 \mathrm{mV} / 1 \mathrm{~cm} / \mathrm{s}$ ) were collected simultaneously with a data acquisition system (PowerLab 8 channel, ADInstruments, Mountain View, CA). Cyclic wave form analysis was performed on the BP data to calculate the MABP (the average amplitude of the BP wave form over one cycle) and systolic and diastolic BP (maximum and minimum values of the BP cycle, respectively). Digital output from the blood gas monitor was converted to an analog signal by a digital-to-analog converter. Fast Fourier analysis was performed on the $\mathrm{CBF}$ velocity signal to determine the systolic, diastolic, and $\mathrm{mCBFv}$.

Autoregulation testing using tracheal suctioning. Adult autoregulation testing procedures, such as altering BP pharmacologically or by rapid removal of a thigh cuff and assessing changes in $\mathrm{CBF}$, are too invasive and may actually induce brain injury in premature infants. Therefore, we tested cerebral autoregulatory capacity by comparing simultaneous changes in $\mathrm{CBF}$ velocity with changes in MABP, during tracheal suctioning procedures. Tracheal suctioning was used as a provocative autoregulatory test since it is known to substantially affect BP, gas exchange, and cerebral hemodynamics in ventilated VLBW infants $(18,19)$

Experimental protocol. Informed consent was obtained from a parent before study participation. Blood pressure, $\mathrm{PaCO}_{2}, \mathrm{PaO}_{2}$, and $\mathrm{CBF}$ velocity were continuously monitored from each VLBW infant before, during, and after clinically indicated tracheal suctioning procedures during the first week of life. Infants were monitored up to twice daily during the first $3 \mathrm{~d}$ and once daily during the next $4 \mathrm{~d}$. Monitoring began approximately 15 min before tracheal suctioning, when infants were quiet and not undergoing any other procedures, and continued for $45 \mathrm{~min}$ postprocedure. The University of Arkansas for Medical Sciences Institutional Review Board approved the study protocol.

Suctioning of ventilated VLBW infants through the endotracheal tube was performed to clear tracheobronchial airways of secretions and to maintain artificial airway patency. This procedure is standardized, was performed only when clinically indicated, and was never routinely scheduled. The endotracheal tube was disconnected from the ventilator and an appropriate sized suction catheter was sterilely inserted to no more than $1 / 2 \mathrm{~cm}$ below the tip of the endotracheal tube. Wall suction $\left(80-100 \mathrm{~cm} \mathrm{H}_{2} \mathrm{O}\right)$ was applied to the catheter, which was withdrawn with one consistent movement. This procedure took 15-20 s. The endotracheal tube was then reconnected to the ventilator with presuction settings and the infant was allowed to recover before the next suction pass. The process was repeated once or twice more until the airway was clear. Saline was rarely used with tracheal suctioning during the first week of life.

Statistical analysis. For the analysis, continuous measurements of MABP (in the presumed autoregulatory plateau range of $30-40 \mathrm{~mm} \mathrm{Hg}$ inclusive) (4), $\mathrm{PaCO}_{2}$, and $\mathrm{mCBFv}$ during and after suctioning procedures during the first postnatal week were used. The objective was to estimate the autoregulatory plateau slope for each infant suctioning session at seven $\mathrm{PaCO}_{2}$ levels $(30,35$, $40,45,50,55$, and $60 \mathrm{~mm} \mathrm{Hg}$ ), chosen to cover the range of mildly hypocapnic and permissively hypercapnic values. Those estimates were then used to test whether the mean autoregulatory plateau slopes at the seven chosen $\mathrm{PaCO}_{2}$ levels were statistically different.

The methodology was as follows:

1. A robust technique (least median of squares) was used to remove data artifacts from each suctioning session (20). The average percentage of movement artifacts was approximately $8 \%$.

2. The following multiple linear regression model was implemented for each suctioning session allowing one to "plug in" different values of $\mathrm{PaCO}_{2}$ $\left(\mathrm{PaCO}_{2}=30,35,40,45,50,55\right.$, and $\left.60 \mathrm{~mm} \mathrm{Hg}\right)$ and obtain autoregulatory plateau slope estimates at each of those levels of $\mathrm{PaCO}_{2}$ : 
$m C B F v=\beta_{0}+\beta_{1} M A B P+\beta_{2} P a C O_{2}+\beta_{3} M A P B^{*} P a C O_{2}+\epsilon$

For example, when $\mathrm{PaCO}_{2}=45 \mathrm{~mm} \mathrm{Hg}$, the autoregulatory plateau slope estimate can be shown to be $\hat{\beta}_{1}+45 \hat{\beta}_{3}$. For statistical modeling validation purposes, the PRESS statistic was compared with the error sum of squares for each model via a percentage change calculation. [Briefly, if the PRESS statistic is close to the error sum of squares, then the fitted model passes validation criteria (21)]. The average percentage change of the PRESS statistic from the error sum of squares was approximately $1.1 \%$.

3. A regression diagnostic, called a leverage ("hat") value, was calculated for each slope estimate from each infant suctioning session. When a leverage value was high, thereby identifying an observation that was the result of extreme extrapolation and therefore statistically unreliable, the corresponding autoregulatory plateau slope estimate was omitted from the analysis.

4. A repeated measures ANOVA was performed on the autoregulatory plateau slope estimates that remained after step 3 (corresponding to acceptable leverage values), to test whether the mean autoregulatory plateau slopes at the seven $\mathrm{PaCO}_{2}$ levels were statistically different. Also, a 95\% confidence interval was constructed for the mean autoregulatory plateau slopes at each of the seven $\mathrm{PaCO}_{2}$ levels. Slopes not statistically different from 0 were considered to represent intact autoregulation, whereas slopes $>0$ represented impaired autoregulation.

\section{RESULTS}

Infant characteristics $(n=43)$ are shown in Table 1. Mean airway pressure was $7.2 \pm 1.5 \mathrm{~cm} \mathrm{H}_{2} \mathrm{O}$ and $\mathrm{Fio}_{2}$ was $26.8 \pm$ 8.1\%. Baseline arterial blood gases were: 1) pH: $7.30 \pm 0.08$; 2) $\mathrm{PaCO}_{2}: 46.2 \pm 7.3 \mathrm{~mm} \mathrm{Hg}$; and 3) $\mathrm{PaO}_{2}: 65.6 \pm 18.5 \mathrm{~mm} \mathrm{Hg}$. MABP was $37.1 \pm 5.2 \mathrm{~mm} \mathrm{Hg}$ and heart rate was $142.5 \pm 13.5$ beats $/ \mathrm{min}$. Mean CBF velocity was $17.6 \pm 5.1 \mathrm{~cm} / \mathrm{s}$. There were $2.7 \pm 1.5$ suctioning sessions per subject. There were 11

Table 1. Characteristics of 43 neonates

\begin{tabular}{lr}
\hline Characteristic & Value \\
\hline Birth weight (g) & $905 \pm 259^{*}$ \\
Gestational age (wk) & $26.9 \pm 2.3^{*}$ \\
Race & $28(65)$ \\
White & $11(26)$ \\
Black & $4(9)$ \\
Hispanic & \\
Gender & $24(56)$ \\
Female & $19(44)$ \\
Male & $41(95)$ \\
Survival & $15(35)$ \\
Multifetal pregnancies & \\
Preterm delivery & $13(30)$ \\
Preterm labor & $10(23)$ \\
Pregnancy-induced hypertension & $8(19)$ \\
Premature rupture of the membranes & $6(14)$ \\
Chorioamnionitis & $3(7)$ \\
Placental abruption or previa & $2(5)$ \\
Prolapsed cord & $1(2)$ \\
Intrauterine death of twin & $40(93)$ \\
Antenatal steroids & $34(79)$ \\
Cesarean section & \\
Apgar scores & $4(1-8) \dagger$ \\
1 Min & $7(1-9) \dagger$ \\
5 Min & \\
Worst cranial ultrasound & $1(2)$ \\
Grade 1 IVH & $1(2)$ \\
Grade 2 IVH & $1(2)$ \\
Grade 3 IVH & $0(0)$ \\
Grade 4 IVH & $1(2)$ \\
Periventricular leukomalacia &
\end{tabular}

Values are no. (\%).

*Plus-minus values are means $\pm \mathrm{SD}$.

$\dagger$ Apgar scores: median (range). suctioning sessions on DOL \#1, 42 on DOL \#2, 28 on DOL \#3, 13 on DOL \#4, 13 on DOL \#5, 7 on DOL \#6, and 3 suctioning sessions on DOL \#7.

Analysis. The effect of increasing $\mathrm{PaCO}_{2}$ on the estimated slopes of the autoregulatory plateau for the study sample is shown in Figure 2 (also see Fig. 1, A and B). Estimated mean slopes and 95\% confidence intervals for $\mathrm{PacO}_{2}$ values 30, 35, $40,45,50,55$, and $60 \mathrm{~mm} \mathrm{Hg}$ are illustrated in Figure 3. The estimated means of the slope of the autoregulatory plateau $\left(\mathrm{cm} / \mathrm{s}^{*} \mathrm{~mm} \mathrm{Hg}{ }^{-1}\right)$ increased as $\mathrm{PacO}_{2}$ increased $(p=0.004)$, and were not affected by day of life $(p=0.994)$. The estimated mean slopes for $\mathrm{PaCO}_{2}$ values of 30,35 , and $40 \mathrm{~mm} \mathrm{Hg}$ represented intact autoregulation as confidence intervals included 0. In contrast, estimated mean slopes for $\mathrm{PaCO}_{2}$ values $\geq 45 \mathrm{~mm} \mathrm{Hg}$ indicated a progressive loss of cerebral autoregulation.

\section{DISCUSSION}

This observational study provides the first data in human VLBW infants during the first week of life suggesting that increasing $\mathrm{PaCO}_{2}$ is associated with progressive impairment of cerebral autoregulation. Detection of autoregulatory capacity during this vulnerable period of life is important because premature infants who lack autoregulation and have perturbations of BP leading to CBF fluctuations are more likely to develop brain injury compared with those with intact CBF regulation $(7,13,14)$. Our observations may be extremely important given the widespread use of permissive hypercapnia in the management of ventilated premature infants throughout the world. However, since we did not examine the relationship between $\mathrm{PaCO}_{2}$ and cerebral autoregulation on neurodevelopmental outcome, we cannot recommend avoidance of permissive hypercapnia at this time.

Intact brain function results from well-regulated CBF mechanisms that are coupled to cerebral metabolic needs and respond appropriately to alterations in $\mathrm{BP}$ and chemical regula-

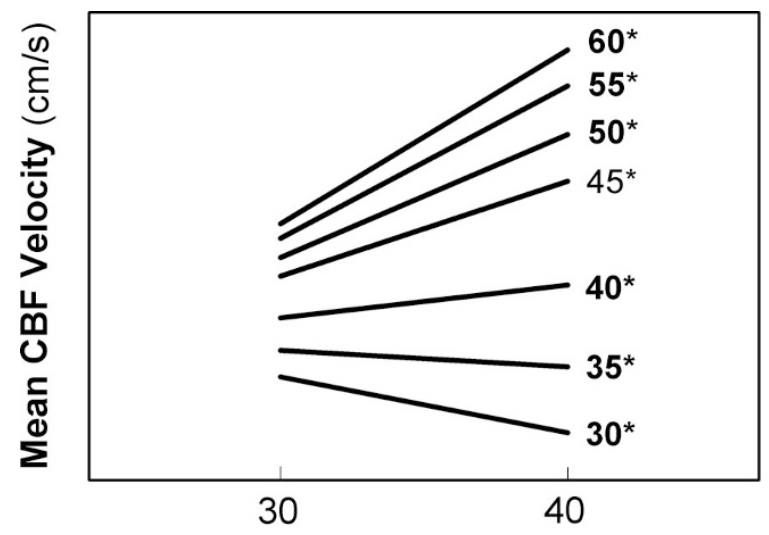

Mean Arterial Blood Pressure $(\mathrm{mm} \mathrm{Hg})$

Figure 2. The influence of increasing $\mathrm{PaCO}_{2}$ values on the estimated slope of the autoregulatory plateau in 43 ventilated VLBW infants. The autoregulatory plateau (the relationship between $\mathrm{mCBFv}$ and MABP at $30-40 \mathrm{~mm} \mathrm{Hg}$ ) is demonstrated for seven $\mathrm{PaCO}_{2}$ * values. A slope near or equal to 0 suggests intact cerebral autoregulation whereas increasing values $>0$ indicate progressively impaired autoregulation. 


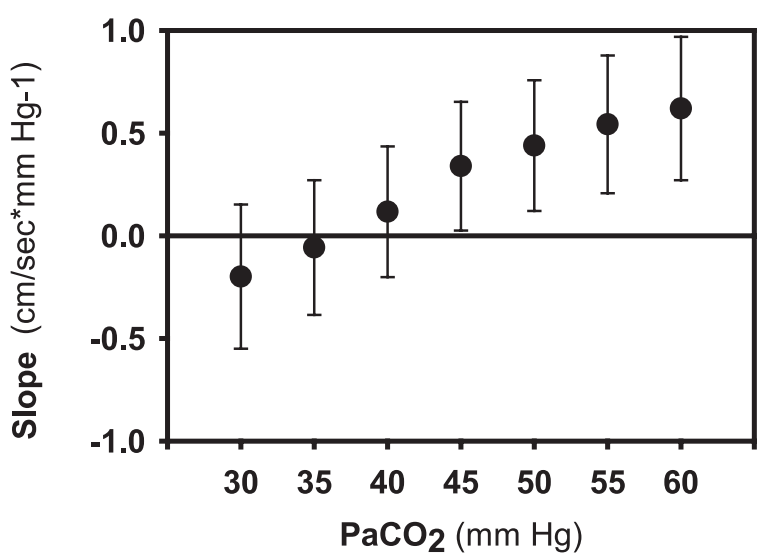

Figure 3. Estimated mean slopes and $95 \%$ confidence intervals of the autoregulatory plateau for $\mathrm{PaCO}_{2}$ values 30, 35, 40, 45, 50, 55, and $60 \mathrm{~mm} \mathrm{Hg}$ for 43 VLBW infants. Bars indicate 95\% confidence intervals for the mean slopes of the autoregulatory plateaus for $\mathrm{PacO}_{2} 30(n=82), 35(n=94), 40(n=$ 100), $45(n=103), 50(n=100), 55(n=90)$, and $60 \mathrm{~mm} \mathrm{Hg}(n=83)$. The horizontal line at slope 0 indicates intact autoregulation. The estimated means of the slope of the autoregulatory plateau $\left(\mathrm{cm} / \mathrm{s} * \mathrm{~mm} \mathrm{Hg}^{-1}\right)$ increased as $\mathrm{PaCO}_{2}$ increased $(p=0.004)$.

tors, such as carbon dioxide, one of the most potent mediators of cerebral vascular tone. Hypercapnia may lead to impaired autoregulation by increasing vasodilation of the cerebral resistance arterioles. Should BP increase concomitantly with hypercapnia, autoregulatory mechanisms may be overcome causing $\mathrm{CBF}$ to increase in a pressure passive manner due to ineffective vasoconstriction. During hypotension, additional vasodilation may not be possible resulting in pressure passive decreases in CBF. Thus, the ability to autoregulate CBF with changes in $\mathrm{BP}$ is significantly dependent on $\mathrm{PaCO}_{2}$ (16).

Our observations in VLBW infants are consistent with classic studies in adults and animals demonstrating that hypercapnia $(>55 \mathrm{~mm} \mathrm{Hg})$ is associated with impaired cerebral autoregulation while moderate hypocapnia and normocapnia is associated with intact autoregulation $(3,15,16,22)$. Lou et al. (23) were the first to suggest that cerebral autoregulation was also similarly affected by $\mathrm{PacO}_{2}$ levels in ventilated premature infants, and that hypocapnia may prevent IVH by reestablishing intact cerebral autoregulation. They examined the relationship between $\mathrm{PaCO}_{2}$ in the first hour of life and subsequent brain injury in VLBW infants and found that those with the highest $\mathrm{PaCO}_{2}$ had the highest incidence of IVH. Thus, prevention of brain injury by restoration of autoregulation by limiting hypercapnia may be more appropriate for managing neonatal respiratory diseases than permissive hypercapnia from a CNS perspective.

There is extensive, albeit retrospective, evidence that hypocapnia in premature infants is associated with poor neurodevelopmental outcome, including periventricular leukomalacia (PVL), IVH, and cerebral palsy (24-26), possibly due to cerebral vasoconstriction, decreased CBF, and decreased cerebral oxygen delivery. Therefore, prevention of hypocapnia must also be a primary objective in the management of these infants. By targeting mild to moderate hypercapnia during ventilation of premature infants, it has been suggested that permissive hypercapnia may be neuroprotective by avoidance of accidental hypocapnia (27). On the other hand, inadvertent hypercapnia may result from this method, and hypercapnia has been associated with IVH $(9,10,12)$. In fact, in the initial pilot trial of permissive hypercapnia in premature infants, many infants in the hypercapnic group had maximum $\mathrm{PaCO}_{2}>55 \mathrm{~mm}$ $\mathrm{Hg}$, perhaps out of the traditional "safe" range of hypercapnia $(1,28)$.

Most of the studies examining the role of cerebral autoregulation in the pathogenesis of neonatal brain injury found that absent autoregulation was associated with IVH whereas intact autoregulation was protective $(7,14,29)$. In a recent study, near-infrared spectroscopy was used to establish the status of autoregulation in ventilated premature infants (7). Eight of 17 infants with impaired autoregulation had severe IVH or PVL. In contrast, only 2 of 15 infants with intact autoregulation suffered severe brain lesions.

Others have attempted to determine the status of cerebral autoregulation in premature infants by examining the slope (percentage change in $\mathrm{CBF} / 1 \mathrm{~mm} \mathrm{Hg}$ change in MABP) of the relationship between measurements of $\mathrm{CBF}$ and MABP $(14,30,31)$. In addition to arbitrarily defining a critical value between 0.5 and $1.5 \%$ change in $\mathrm{mCBFv} / 1 \mathrm{~mm} \mathrm{Hg}$ change in MABP as intact autoregulation (30-32), they did not take into account simultaneous changes in $\mathrm{PaCO}_{2}$ that clearly cause changes in $\mathrm{CBF}$ and MABP in the same direction, which may be interpreted as impaired autoregulation. Although these critical values are somewhat arbitrary, values as high as $2.7-4 \%$ were present in infants with severe IVH, and presumably represented impaired autoregulation $(14,32)$. We do not report a critical value inasmuch as we do not have enough study infants with severe brain injury to define a specific value. We have, however, shown that increasing slopes are associated with impaired autoregulation and speculate that premature infants with increased slopes are at increased risk for brain injury.

There were four limitations of this study. Even though we used Doppler ultrasound to measure $\mathrm{mCBFv}$ instead of direct measures of CBF, neonatal (33) studies found good correlation between relative changes in both measurements. The Doppler method has the advantages of being noninvasive, easily performed at the bedside, and providing real-time information. Further, it does not require radioactive materials, patient manipulations, or alterations of $\mathrm{Fio}_{2}$. The second limitation is that we used statistical modeling to determine the effects of $\mathrm{PaCO}_{2}$ on cerebral autoregulation instead of prospectively assigning infants to certain $\mathrm{PaCO}_{2}$ ranges and comparing their capacity for autoregulation. Thus, we are cautious in making sweeping recommendations regarding the continued use of permissive hypercapnia in ventilated premature infants from our observational trial. Thirdly, while we observed that increasing $\mathrm{PaCO}_{2}$ was associated with impaired autoregulation, we did not directly demonstrate that impaired autoregulation was associated with brain injury. This was not possible given the lack of brain injury in our cohort of infants, although there is sufficient previous evidence in the neonatal literature demonstrating that impaired autoregulation is associated with brain injury. Lastly, we did not examine the effects of other variables such as gestational age, postnatal age, mode of ventilation, ductal 
shunting, requirement for vasopressor support, and interactions between the variables, on the capacity for autoregulation. Our goal in this manuscript, however, was to examine the effects of hypercapnia on the capacity for autoregulation. Additional research and study subjects are required to assess other factors that may be associated with autoregulatory capacity in VLBW infants.

In conclusion, the slope of the autoregulatory plateau increases with increasing $\mathrm{PaCO}_{2}$, suggesting the cerebral circulation becomes more pressure passive with increasing hypercapnia. These novel data on the effects of elevated $\mathrm{PaCO}_{2}$ on the capacity for cerebral autoregulation raise concerns regarding the widespread use of permissive hypercapnia in VLBW infants. Careful control of $\mathrm{PaCO}_{2}$ during the first week of life, by preventing hypercapnia as well as hypocapnia, may be identified as a potential clinical goal (by using a continuous arterial blood gas system) to restore intact cerebral autoregulation, prevent the initiation or extension of IVH, and prevent the development of PVL in ventilated VLBW infants.

Acknowledgments. The authors thank Natalie C. Sikes, Melanie Mason, Rachel Choate, Tammy Drake, Phyllis Mayfield, and Jennifer Simmons; and the University of Arkansas for Medical Sciences neonatologists, NICU nurses, respiratory therapists, and ultrasound technicians. We also thank Gerald A. Dienel, Ph.D., Jeffrey M. Perlman, M.D., K.J.S. Anand, MBBS, D.Phil., and Richard W. Hall, M.D., for reviewing the manuscript.

\section{REFERENCES}

1. Mariani G, Cifuentes J, Carlo WA 1999 Randomized trial of permissive hypercapnia in preterm infants. Pediatrics 104:1082-1088

2. Carlo WA, Stark AR, Wright LL, Tyson JE, Papile LA, Shankaran S, Donovan EF, Oh W, Bauer CR, Saha S, Poole WK, Stoll B 2002 Minimal ventilation to prevent bronchopulmonary dysplasia in extremely-low-birth-weight infants. $J$ Pediatr 141:370-375.

3. Harper AM 1966 Autoregulation of cerebral blood flow: influence of the arterial blood pressure on the blood flow through the cerebral cortex. J Neurol Neurosurg Psychiatry 29:398-403

4. van de Bor M, Walther FJ 1991 Cerebral blood flow velocity regulation in preterm infants. Biol Neonate 59:329-335

5. Papile LA, Rudolph AM, Heymann MA 1985 Autoregulation of cerebral blood flow in the preterm fetal lamb. Pediatr Res 19:159-161

6. Lou HC, Lassen NA, Friis-Hansen B 1979 Impaired autoregulation of cerebral blood flow in the distressed newborn infant. $J$ Pediatr 94:118-121

7. Tsuji M, Saul JP, du Plessis A, Eichenwald E, Sobh J, Crocker R, Volpe JJ 2000 Cerebral intravascular oxygenation correlates with mean arterial pressure in critically ill premature infants. Pediatrics 106:625-632

8. Kaiser JR, Gauss CH, Williams DK 2004 Surfactant administration acutely affect cerebral and systemic hemodynamics and gas exchange in very-low-birth-weight infants. J Pediatr 144:809-814
9. Levene MI, Fawer CL, Lamont RF 1982 Risk factors in the development of intraventricular haemorrhage in the preterm neonate. Arch Dis Child 57:410-417

10. van de Bor M, van Bel F, Lineman R, Ruys JH 1986 Perinatal factors and periventricular-intraventricular hemorrhage in preterm infants. Am J Dis Child 140:1125-1130

11. Skouteli HN, Kuban KC, Leviton A, Brown ER, Krishnamoorthy KS, Pagano M, Allred EN, Sullivan KF, Baglivo JA, Huff KR, Epstein MF 1988 Arterial blood gas derangements associated with death and intracranial hemorrhage in premature babies. J Perinatol 8:336-341

12. Wallin LA, Rosenfeld CR, Laptook AR, Maravilla AM, Strand C, Campbell N, Dowling S, Lasky RE 1990 Neonatal intracranial hemorrhage: II. Risk factor analysis in an inborn population. Early Hum Dev 23:129-137

13. Perlman JM, Goodman S, Kreusser KL, Volpe JJ 1985 Reduction in intraventricular hemorrhage by elimination of fluctuating cerebral blood-flow velocity in preterm infants with respiratory distress syndrome. $N$ Engl J Med 312:1353-1357

14. Pryds O, Greisen G, Lou H, Friis-Hansen B 1989 Heterogeneity of cerebral vasoreactivity in preterm infants supported by mechanical ventilation. J Pediatr 115:638645

15. Häggendal E, Johansson B 1965 Effects of arterial carbon dioxide tension and oxygen saturation on cerebral blood flow autoregulation in dogs. Acta Physiol Scand Suppl 258:27-53

16. Hauerberg J, Juhler M, Rasmussen G 1993 Cerebral blood flow autoregulation after experimental subarachnoid hemorrhage during hyperventilation in rats. $J$ Neurosurg Anesthesiol 5:258-263

17. American Institute of Ultrasound in Medicine 1993 Bioeffects and Safety of Diagnostic Ultrasound. American Institute of Ultrasound in Medicine, Laurel, MD, pp $80-86$

18. Perlman JM, Volpe JJ 1983 Suctioning in the preterm infant: effects on cerebral blood flow velocity, intracranial pressure, and arterial blood pressure. Pediatrics 72:329334

19. Skov L, Ryding J, Pryds O, Greisen G 1992 Changes in cerebral oxygenation and cerebral blood volume during endotracheal suctioning in ventilated neonates. Acto Paediatr 81:389-393

20. Rousseeuw P, Leroy A 1987 Robust Regression and Outlier Detection. John Wiley \& Sons Inc., New York, pp 1-157

21. Neter J, Kutner M, Nachtsheim C, Wasserman W 1996 Applied Linear Statistical Models, pp 1-439. WCB/McGraw-Hill, Boston

22. Aaslid R, Lindegaard KF, Sorteberg W, Nornes H 1989 Cerebral autoregulation dynamics in humans. Stroke 20:45-52

23. Lou HC, Phibbs RH, Wilson SL, Gregory GA 1982 Hyperventilation at birth may prevent early periventricular haemorrhage. Lancet 1:1407

24. Greisen G, Munck H, Lou H 1987 Severe hypocarbia in preterm infants and neurodevelopmental deficit. Acta Paediatr Scand 76:401-404

25. Okumura A, Hayakawa F, Kato T, Itomi K, Maruyama K, Ishihara N, Kubota T, Suzuki M, Sato Y, Kuno K, Watanabe K 2001 Hypocarbia in preterm infants with periventricular leukomalacia: The relation between hypocarbia and mechanical ventilation. Pediatrics 107:469-475

26. Collins MP, Lorenz JM, Jetton JR, Paneth N 2001 Hypocapnia and other ventilationrelated risk factors for cerebral palsy in low birth weight infants. Pediatr Res 50:712-719

27. Thome UH, Carlo WA 2002 Permissive hypercapnia. Semin Neonatol 7:409-419

28. Ambalavanan N, Carlo WA 2001 Hypocapnia and hypercapnia in respiratory management of newborn infants. Clin Perinatol 28:517-531

29. Rosenkrantz TS, Diana D, Munson J 1988 Regulation of cerebral blood flow velocity in nonasphyxiated, very low birth weight infants with hyaline membrane disease. $J$ Perinatol 8:303-308

30. Jorch G, Jorch N 1987 Failure of autoregulation of cerebral blood flow in neonates studied by pulsed Doppler ultrasound of the internal carotid artery. Eur J Pediatr 146:468-472

31. Panerai RB, Kelsall AW, Rennie JM, Evans DH 1995 Cerebral autoregulation dynamics in premature newborns. Stroke 26:74-80

32. Menke J, Michel E, Rabe H, Bresser BW, Grohs B, Schmitt RM, Jorch G 1993 Simultaneous influence of blood pressure, $\mathrm{PCO}_{2}$, and $\mathrm{PO}_{2}$ on cerebral blood flow velocity in preterm infants of less than 33 weeks' gestation. Pediatr Res 34:173-177

33. Greisen G, Johansen K, Ellison PH, Fredriksen PS, Mali J, Friis-Hansen B 1984 Cerebral blood flow in the newborn infant: Comparison of Doppler ultrasound and ${ }^{133}$ xenon clearance. J Pediatr 104:411-418 